\title{
Esophageal Adenoid Cystic Carcinoma
}

National Cancer Institute

\section{Source}

National Cancer Institute. Esophageal Adenoid Cystic Carcinoma. NCI Thesaurus. Code C5342.

An infrequent esophageal carcinoma arising from esophageal glands. (WHO) 